\title{
NEWS AUS DEM BEREICH DER BIBLIOTHEKARISCHEN AUSBILDUNG IN ÖSTERREICH IM JAHR 2017 UND 2018
}

\author{
von Gabriele Pum
}

Zusammenfassung: Dieser Beitrag bietet einen Überblick über die Ausbildung im wissenschaftlichen Bibliothekswesen Österreichs in den Jahren 2017 und 2018.

Schlüsselwörter: Universität Wien; Universität Innsbruck; Österreichische Nationalbibliothek; Interuniversitärer Universitätslehrgang Library and Information Studies; Grundlehrgang; Masterlehrgang; Archiv-, Bibliotheks- und Informationsassistent/In; Lehrlinge; Mittlere Qualifikation; Ausbildungslehrgang „Bibliothek, Information und Dokumentation"; Marianne-Englert-Preis; Ausbildungsprojekte; Zertifikatskurse; 2017; 2018

\section{NEWS FROM THE FIELD OF EDUCATION AND TRAINING OF LIBRARIANS IN AUSTRIA 2017 AND 2018}

Abstract: A report about continuing education and training programs in Library and Information Studies in the years 2017 and 2018 in Austria.

Keywords: University of Vienna; University of Innsbruck; Austrian National Library; Cooperation; University course „Library and Information Studies"; Basic training course; Advanced training course; Apprenticeship „Archives; Library and Information assistant"; Continuing education; Projects; Marianne-Englert-Award; Certificate courses; 2017; 2018

DOI: https://doi.org/10.31263/voebm.v71i3-4.2163

(C) Gabriele Pum

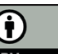

Dieses Werk ist lizenziert unter einer

Creative-Commons-Lizenz Namensnennung 4.0 International 


\section{Universitätslehrgang Library and Information Studies: Grundlehrgang}

Als einziger Standort in Österreich startete im WiSe 2016/17 an der Österreichischen Nationalbibliothek der 13. Grundlehrgang des Interuniversitären Universitätslehrgangs Library and Information Studies mit 22 Studierenden (davon 18 AkademikerInnen, 16 Studentinnen und 6 Studenten).

Im Rahmen des Lehrgangs ist die Erstellung von Projektarbeiten vorgesehen, die jeweils in Gruppen von zwei bis vier Studierenden durchgeführt werden. Folgende Abschlussprojekte entstanden im Rahmen des Lehrganges 2016/2017:

- Archivsystem historischer Wetterkarten

- Reorganisierung der Fachbibliothek des Bibliotheksverbandes Österreich

- DLOÖ-Crowdsourcing. Weiterentwicklung - Usability

- Erschließung Teilvorlass von Walter Kappacher am Literaturarchiv der ÖNB

- Teilbearbeitung des Nachlasses von Tobi Reiser (d. Ä.) in der Bibliothek des Salzburg Museum

- „Grauware“: Kleinschriften, Sonderdrucke, Predigten in Stift Heiligenkreuz

- Erschließung und Aufarbeitung der Forschungsbibliothek des Exegeten P. Nivard Schlögl OCist (+1939) in Stift Heiligenkreuz

- Detailerschließung des Fotoarchivs der Freien Österreichischen Jugend (FÖJ)

- Erweiterung eines Bibliotheksbestandes um E-Books: Angebot und Nutzung von englisch-sprachigen E-books an der Modul University

- Analyse\#NewPictures (Multimediales ORF-Archiv)

Erstmals haben die Studierenden in Eigeninitiative eine Bibliotheksreise nach Rom initiiert, die unter anderem auch in die Biblioteca Nazionale und natürlich als Highlight der Reise in das Vatikanische Archiv und die Vatikanische Bibliothek führten.

Einer Absolventin dieses Lehrgangs wurde der Literaturpreis Alpha 2016 der Casinos Austria verliehen und das Projektteam \#NewPictures erhielt den internationalen Marianne-Englert-Preis des Vereins für Medieninformation und Mediendokumentation, der am 10. April 2018 in Bonn überreicht wurde.

Im Jänner 2017 übersiedelte das Ausbildungszentrum der Österreichischen Nationalbibliothek für die Dauer der Renovierungsarbeiten des Parlaments an den neuen temporären Standort des Ausbildungszentrums in die Augasse 2-6 (9. Bezirk) in das alte ÖH-Gebäude der ehemaligen Wirtschaftsuniversität Wien.

Im WS 2017/18 startete der 14. Grundlehrgang des Interuniversitären Universitätslehrgangs Library and Information Studies an der Universitätsbibliothek Wien mit 24 Studierenden und an der Österreichischen Nati- 
onalbibliothek mit 22 Studierenden. Inhaltlich geprägt ist der Lehrgang durch den Umstieg auf das neue Bibliotheksverbundsystem ALMA. Um die Studierenden für alle Anforderungen des Arbeitsmarkts zu qualifizieren, wird die Möglichkeit geboten, sowohl im alten als auch neuen Katalogisierungs- und Bibliothekssystemen ausgebildet zu werden. Neu ist auch, dass die Studierenden von der Vizepräsidentin der VÖB und Leiterin der Arbeitsgruppe Prekäre Arbeitsverhältnisse im Workshop Berufseinstieg und praktische Bewerbungstipps wertvolle Hinweise für Ihren Karrierestart erhalten.

Folgende Abschlussprojekte entstanden im Rahmen des Lehrganges an der Österreichischen Nationalbibliothek:

- Wetter und Leben

- Neuorganisation der Bibliothek des Vereins Selbstlaut

- Patentlösungen - zur Neuausrichtung der Bibliothek des Österreichischen Patentamtes

- Ernst Aloysius Kein (1928-1985). Feinerschließung des Nachlasses Literaturarchiv der Österreichischen Nationalbibliothek

- Provenienzerschließung der Bibliotheca Arresiana

- E-Bibliothek Politische Bildung und Menschenrechtsbildung. Erarbeitung eines Konzeptes für das Ludwig Boltzmann Institut für Menschenrechte

- Depotprojekt Heiligenkreuz: Sichtbarmachung eines historischen Bestandes in der Stiftsbibliothek

Folgende Abschlussprojekte entstanden im Rahmen des Lehrganges an der Universitätsbibliothek Wien:

- Falknereiarchiv: Der Bestand des Archives (Schloss Waldreichs im Waldviertel) in einer entsprechenden Bibliothekssoftware erfassen und katalogisieren

- Her Majesty's Overseas Civil Service - Sammlung OSPA Journals

- Ethnographische Datenarchivierung

- Bearbeitung der Oberin-Gleixner-Bibliothek

- Das „Inventarium der Bibliothek im k.k. Thierarzney-Institute“. Ein medizinisches Bücherverzeichnis des Vormärz

- Anreichung einer Bibliographie mit Bibliometrie - Fallstudie am Beispiel von Anestis Logothetis

- Erstellen eines Internet-Tools zur Darstellung und Verknüpfung von bibliographischen Informationsquellen

- Formate für die langfristige Verfügbarkeit von digitalen Objekten

- Die wissenschaftliche Bibliothek tagt - Tagung und die Herausgabe des Tagungsbandes „Treuhänderische Übernahme und Verwarung - interdisziplinär und international betrachtet" 
Im WS 2018/19 startete der fünfzehnte Grundlehrgang des Interuniversitären Universitätslehrgangs Library and Information Studies an der Universitätsbibliothek Wien mit 26 Studierenden und der Österreichischen Nationalbibliothek mit 22 Studierenden.

\section{Universitätslehrgang Library and Information Studies: Masterlehrgang}

2016 endeten die Theorieteile des ersten Masterlehrgangs Library and Information Studies nach Curriculum 2013 der Universität Wien, der in Kooperation mit der Österreichischen Nationalbibliothek durchgeführt wurde. Die 24 Studierenden wählten sieben von neun möglichen Wahlfächern. Das Hauptinteresse lag vor allem auf betriebswirtschaftlichen Kenntnissen, Führungs- und Managementaufgaben und der Auseinandersetzung mit neuen Trends in der Informationstechnologie und im Informations- und Wissensmanagement, die das Bibliothekswesen betreffen. Methoden und Konzepte werden in der „Managementfabrik“ im Rahmen eines Planspieles erprobt und die theoretisch vermittelten Inhalte selbständig in der „eigenen Bibliothek" angewandt, wobei auch die Konsequenzen der Entscheidungen analysiert wurden. Dieses spannende Projekt begleitet die Studierenden durch den gesamten Masterlehrgang, dessen Theorieteile im Jahr 2016 abgeschlossen wurden. Großer Wert wurde bei der Konzeption des neuen Curriculums sowohl auf die Reflexion der eigenen Arbeitssituation als auch auf den Praxistransfer in allen Modulen gelegt.

Sieben KollegInnen beendeten 2017 den Masterlehrgang mit ihren Masterthesen, die sich mit Themen aus den Bereichen Requirements Engineering, Publikationsverhalten, Digitale Ressourcen und dem Öffentlichen Bibliothekswesen auseinandersetzten. Die entsprechenden Abstracts wurden in den Mitteilungen der VÖB 3/4 (2017) veröffentlicht. ${ }^{1}$

\section{Abstracts 2018}

Mag. ${ }^{a}$ Michaela Mayr: Crowdsourcing für Bibliotheken Best Practices und Handlungsempfehlungen

Gedächtnisinstitutionen wie Bibliotheken sind verantwortlich für die Sammlung, Bewahrung, Erschließung und Zugänglichmachung des kulturellen Erbes. Nach einer Phase langjähriger und umfangreicher Digitalisierungsprojekte sollen neben der reinen Betrachtungsmöglichkeit digitaler Objekte neue partizipative Zugänge zu Beständen geschaffen werden. Die 
Entwicklung des Webs vom Publikations- zum Interaktionsmedium (auch bekannt als Web 2.0) gab Mitte der 2000er Jahre den Impuls für die Entstehung von Crowdsourcing-Projekten. Dabei werden Aufgaben - meist als Online-Aktivität - an eine Gruppe von Individuen („crowd“) ausgelagert („,sourcing“). Das Potential für Bibliotheken ist groß: Man kann Benutzerlnnen stärker einbinden und sich das Wissen der Masse zu Nutze machen. Das Engagement in derartigen Projekten betrifft die strategische Ausrichtung der Bibliothek und bedeutet für viele Institutionen einen Kulturwandel. Vor Beginn des Engagements müssen Chancen und Risiken sorgfältig abgewogen werden, dann gilt es, zahlreiche Entscheidungen zur Ausgestaltung zu treffen. Überlegt werden muss dabei, welche Art von Crowdsourcing genutzt werden soll, wie der Crowdsourcing-Prozess gestaltet werden kann, welche Plattformen geeignet sind und wie wichtige Erfolgsfaktoren wie Motivation und Qualität bei eigenen Crowdsourcing-Projekten berücksichtigt werden können.

Ein großer Teil dieser Arbeit widmet sich der Analyse von Best Practice-Beispielen und ihren Elementen. Ausgewählt wurden Initiativen aus dem GLAM-Sektor (galleries, libraries, archives, museums) und aus dem Bereich Citizen Science, denen eine wissenschaftliche Zielsetzung zugrunde liegt. Daraus abgeleitet werden Lessons Learned und Handlungsempfehlungen. Weiters bietet die Arbeit praktische und theoretische Hinweise zum Projektmanagement eines Crowdsourcing-Projekts in Bibliotheken.

\section{Mag. Márton Villányi: Lizenzverträge mit Open-Access-Komponenten an öster- reichischen Bibliotheken}

Konsortiale Subskriptionsverträge zwischen Verlagen und wissenschaftlichen Bibliotheken regeln den digitalen Zugang zu Publikationen. Seit einigen Jahren verstärkt sich jedoch die Tendenz hin zu einem frei zugänglichen Publikationswesen (Open Access). Als Konsequenz dieser Entwicklung verändern sich allmählich auch die Vertragsverhältnisse zwischen Lizenzgebern und -nehmern: Immer mehr Vereinbarungen nehmen nun auch auf das Open-Access-Publizieren Einfluss.

Die vorliegende Arbeit versucht, die verschiedenen Zugänge solcher konsortialen Lizenzvereinbarungen mit Open-Access-Komponenten anhand der in Österreich abgeschlossenen Verträge gegenüberzustellen. So werden die Unterschiede zwischen reinen Subskriptionsverträgen und diversen neuartigen Vereinbarungen mit Open-Access-Komponenten beschrieben. Dabei wird die Notwendigkeit von modifizierten Evaluierungs- 
methoden bei der Beurteilung von diesen neuen Lizenzvereinbarungen ersichtlich. Als wesentlichstes neues Element solcher Analysen wird die Evaluierung von Open-Access-Publikationszahlen identifiziert. In der Arbeit wird nun der Versuch unternommen, diese Publikationsanalysen für die bestehenden Open-Access-Vereinbarungen durchzuführen. Der Fokus liegt dabei auf quantitativen Fragen: Wie entwickeln sich die Publikationszahlen? Wie verändern sich die Open-Access-Anteile? Die Datengrundlage bilden Publikationsmeldungen von den Verlagen und Abfragen aus der Datenbank Scopus. Die gegenübergestellten Ergebnisse zeigen, dass die unterschiedliche Konzeption der Verträge auch stark divergierende Ergebnisse erzielen: So kann der Schwerpunkt der einzelnen Verträge auf Kostenersparnisse oder aber auf die effiziente Steigerung der Open-AccessRaten gelegt werden.

Es ist davon auszugehen, dass in den folgenden Jahren zahlreiche weitere konsortiale Open-Access-Verträge verhandelt werden. Die hier vorliegenden Erkenntnisse können dabei als Orientierungshilfe herangezogen werden.

\section{Mag. ${ }^{a}$ Lisa Weinberger: From service to partnership - Die Embedded Library als Konzept für Unternehmensbibliotheken}

Die vorliegende Arbeit beschäftigt sich mit dem Konzept Embedded Librarian bezogen auf Unternehmensbibliotheken. Bei diesem Managementkonzept werden Bibliothekare und Bibliothekarinnen direkt in die Kundengruppe integriert. Insbesondere Unternehmensbibliotheken arbeiten eng mit ihrer Kundengruppe zusammen und sind meist sowohl physisch als auch organisatorisch in diese eingebunden. Ziel der Arbeit ist es anhand ausgewählter Literatur darzustellen, welche Vorteile die Einbindung von Informationsexperten und Informationsexpertinnen innerhalb einer Kundengruppe hat und wie es gelingt von einer reinen Serviceposition, zu einer gleichwertigen Partnerschaft aufzusteigen.

Durch einen theoretischen Überblick über die Geschichte und Aufgaben von Unternehmensbibliotheken, sowohl im deutschsprachigen als auch im anglo-sächsischen Raum, werden die Besonderheiten von Unternehmensbibliotheken genauer erläutert.

Ein weiterer Aspekt ist die Einführung in das Konzept der Embedded Library. Durch Fallbeispiele wird dargestellt, in welcher Form sich Bibliothekare und Bibliothekarinnen in die Kundengruppe einbinden können. Ein Exkurs über One-Person Librarians gibt Einblicke in die Herausforderungen vieler Unternehmensbibliotheken und zeigt die Überschneidungen 
zwischen One-Person Librarians und Embedded Librarians auf. Durch das Embedded Librarian Konzept entsteht ein neues Selbstverständnis von Bibliothekare und Bibliothekarinnen und für deren Aufgabenbereiche innerhalb der Trägerorganisation. Durch eine erfolgreiche Umsetzung des Embedded Librarian-Konzepts können Informationsexperten und Informationsexpertinnen eine erfolgreiche und langfristige Zusammenarbeit mit dem Unternehmen eingehen.

\section{Mittlere Qualifikation}

Die Universitäten Wien und Innsbruck führen in Kooperation den Ausbildungslehrgang Bibliothek, Information und Dokumentation für die Tätigkeitsbereiche mittlerer Qualifikation durch.

Der Lehrgang startete im Oktober 2017 und endete im April 2018. Die insgesamt sechs Module fanden abwechselnd in Wien und Innsbruck statt. 13 Bibliothekarlnnen aus Wien, Innsbruck, Graz und Leoben nahmen an dem Lehrgang teil.

\section{Lehrberuf: Archiv-, Bibliotheks- und Informationsassistent/In}

Am 4. und 5. Juli sowie am 2. Oktober 2018 absolvierten 28 Lehrlinge ihre Lehrabschlussprüfung im Gewerbehaus der Wirtschaftskammer in Wien.

Am 25./26. Juni und 21. September 2018 traten 22 Lehrlinge zu ihrer Lehrabschlussprüfung im Gewerbehaus der Wirtschaftskammer an.

\begin{tabular}{|c|l|l|l|l|}
\hline Jahr & $\begin{array}{c}\text { Universitäts- } \\
\text { lehrgang Library } \\
\text { and Information } \\
\text { Studies: } \\
\text { Grundlehrgang }\end{array}$ & $\begin{array}{c}\text { Universitäts- } \\
\text { lehrgang Library } \\
\text { and Information } \\
\text { Studies: } \\
\text { Masterlehrgang }\end{array}$ & $\begin{array}{c}\text { Mittlere } \\
\text { Qualifikation }\end{array}$ & $\begin{array}{c}\text { Lehrberuf: } \\
\text { Archiv-, Biblio- } \\
\text { theks- und } \\
\text { Informations- } \\
\text { assistent/in }\end{array}$ \\
\hline 2017 & $\begin{array}{l}22 \text { Informations- } \\
\text { expertInnen }\end{array}$ & $\begin{array}{l}7 \text { Masters of } \\
\text { Science }\end{array}$ & $\begin{array}{l}13 \text { Absolvent- } \\
\text { Innen }\end{array}$ & $\begin{array}{l}28 \mathrm{ABI} \text {-Assistent- } \\
\text { Innen }\end{array}$ \\
\hline 2018 & $\begin{array}{l}45 \text { Informations- } \\
\text { expertInnen }\end{array}$ & $\begin{array}{l}3 \text { Masters of } \\
\text { Science }\end{array}$ & $\begin{array}{l}22 \mathrm{ABI} \text {-Assistent- } \\
\text { Innen }\end{array}$ \\
\hline
\end{tabular}

Tab. 1: Statistik der Absolventinnnen und Absolventen der Bibliotheksausbildung in Österreich 2017 


\section{Zertifikatskurse}

Der Zertifikatskurs „Bestandsmanagement in Bibliotheken und Informationseinrichtungen" richtet sich an Bibliotheks- und InformationsspezialistInnen, IT- und MedienspezialistInnen, sowie Personen, die im Bereich forschungsunterstützender Services bereits tätig sind oder sich einen Überblick über die Fragestellungen und Services in diesem Bereich verschaffen wollen. Zehn Teilnehmerlnnen absolvierten die Module Bestandsmanagement, Optimierung von Bibliotheksservices, Wissenschaftliches Publizieren und Langzeitarchivierung.

Weitere Informationen sowie die Links zu den einzelnen Ausbildungsstandorten zur Ausbildung in Österreich finden Sie auf der Homepage der Arbeitsgemeinschaft der Ausbildungsverantwortlichen Österreichs für das Bibliothekspersonal der Universitäten: http://www.bibliotheksausbildung.at.

Dr. ${ }^{\text {in }}$ Gabriele Pum Österreichische Nationalbibliothek E-Mail: gabriele.pum@onb.ac.at

1 Gabriele Pum (2017): Abstracts der Masterthesen des Interuniversitären Universitätslehrganges Library and Information Studies 2017. Mitteilungen der Vereinigung Österreichischer Bibliothekarinnen und Bibliothekare 70(3/4), S. 357-363. https://doi.org/10.31263/voebm. v70i3.1958 